\title{
Evaluación de la tenacidad en el hormigón proyectado reforzado con fibras poliméricas de alto módulo
}

\author{
T. GARCIA, L. AGULLÓ, A. AGUADO, G. CANALS \\ E.T.S. Ingenieros de Caminos, Canales y Puertos de Barcelona, Universitat Politècnica de Catalunya. Barcelona. España
}

\begin{abstract}
El presente trabajo describe la metodología seguida y los resultados obtenidos en un estudio relativo a la evaluación de la tenacidad del hormigón proyectado con fibras poliméricas de alto módulo según dos procedimientos de ensayo distintos: el Plate Bending Test de la norma europea EFNARC de configuración hiperestática; y el Round Determinate Panel Test, ensayo de tipo isostático propuesto por E.S. Bernard. Asimismo, se ha ensayado hormigón de referencia sin fibras y con fibras metálicas. El estudio se ha completado con la caracterización del material, comprobando las modificaciones en las características del hormigón por la incorporación de las fibras. Desde el punto de vista del control de calidad se han contrastado ambos ensayos con el fin de determinar el ensayo que mejor se adapta a este tipo de aplicaciones.
\end{abstract}

Palabras clave: Hormigón proyectado, fibras, tenacidad, Plate Bending Test, Round Determinate Test.

\section{Toughness evaluation of shotcrete reinforced with high modulus polymeric fibers}

This article describes the methodology and presents results of a study of the evaluation of the post-cracking performance of shotcrete reinforced with high modulus polymeric fibers using two panel-based tests: the Plate Bending Test of EFNARC, and the Round Determinate Panel Test proposed by Bernard. A plain shotcrete and a steel fiber reinforced shotcrete have also been tested for comparison. The material properties in each case have also been characterized. An evaluation of the two panel tests has been performed considering the requirements of shotcrete applications.

Key words: Shotcrete, fiber, toughness, Plate Bending Test, Round Determinate Test.

\section{INTRODUCCIÓN}

La proyección es un sistema de puesta en obra del hormigón de elevada producción al englobar en una sola actuación, la proyección del material a gran velocidad sobre un soporte, dos etapas del hormigonado tradicional, la colocación y compactación.

Es conocido que la incorporación de fibras en el hormigón mejora distintas vertientes de su comportamiento como material estructural en lo que se refiere, fundamentalmente, a la fisuración, tenacidad y resistencia al impacto (1). En este sentido, la incorporación de fibras en la proyección es interesante desde el punto de vista de las grandes deformaciones a las que puede estar sometido el hormigón en determinadas aplicaciones (p.e. sostenimiento de túneles, estabilización de taludes, otras) (3). Desde el punto de vista constructivo, el uso de fibras incrementa el rendimiento y mejora la seguridad en obra, al reducir las operaciones de colocación de malla, y reduce costes al no requerir mayor espesor de capa en aquellas zonas donde la malla no se ajuste al soporte.

Son varias las fibras existentes en el mercado, pero destacan por su mayor consumo, y en este orden, las fibras de acero, de polipropileno, de vidrio y poliester. Las fibras sintéticas presentan diversas ventajas frente a las fibras de acero: son químicamente inertes, ligeras y de menor coste. No obstante, este tipo de fibras no está exento de algunos inconvenientes: baja adherencia entre fibra y matriz y menor módulo de elasticidad. A tal efecto han aparecido en el mercado un nuevo tipo de fibras poliméricas estructurales, constituidas por una mezcla de polipropileno y polietileno, que disponen de un módulo de elasticidad mayor que sus antecesoras.

Desde el punto de vista del control de calidad de los hormigones reforzados con fibras existen diferentes tipos de ensayo para valorar el comportamiento a tenacidad; entre éstos podemos distinguir dos grupos: los ensayos de viga, en los que una probeta prismática se en- saya unidireccionalmente a flexotracción; y los ensayos de placa, que permiten evaluar la capacidad de las fibras en las dos direcciones del plano (1) (2) (3). De los ensayos de placa destacan por su mayor difusión el ensayo de la norma europea EFNARC (European Federation of National Associations of Specialist Contractors and Material Suppliers for the Construction Industry), Plate Bending Test (4), y el Round Determinate Panel Test desarrollado por el profesor E.S. Bernard de la Universidad of Western Sydney (Nepean, Australia) (5). La diferencia entre ambos ensayos radica en la redistribución de esfuerzos que permite el ensayo EFNARC.

El presente trabajo se ha centrado en el estudio de las propiedades del hormigón proyectado reforzado con fibras poliméricas de alto módulo (HPRFP), concretamente en como afecta a las propiedades del hormigón fresco y endurecido (resistencias, densidad, porosidad, etc.) la incorporación de las fibras, profundizando en el estudio de la tenacidad a flexotracción, es decir, de la capacidad de absorción de energía después de la fisuración del hormigón contrastando los dos ensayos de placa más generalizados.

\section{CAMPAÑA EXPERIMENTAL}

\subsection{Dosificación y fabricación}

Se han proyectado tres hormigones: un hormigón control sin fibras (HPC); un hormigón reforzado con fibras metálicas (HPRFM) de tipo "hooked end" (ver figura 1), es decir, con patas de anclaje; y un hormigón reforzado con fibras poliméricas de alto módulo (HPRFP). En la Tabla I se detallan las características de ambas fibras. En todas las mezclas se ha mantenido la dosificación de referencia, obtenida a 
partir de la propuesta metodológica desarrollada por Rodríguez (6) para dosificación de hormigones proyectados y que se detalla en la Tabla II, variando únicamente la cantidad de fibra. Con el objeto de tener hormigones similares se solicitaron a los fabricantes las cantidades de fibra a incorporar necesarias para obtener una capacidad de absorción de energía en ensayo de placa EFNARC clase B (entre 650 y 750 Joules).

TABLA I. CARACTERÍSTICAS DE LAS FibRAS.

\begin{tabular}{|l|l|l|}
\hline Característica & Fibra metálica & Fibra polimérica \\
\hline Materia prima & Acero & Polipropileno y polietileno \\
\hline Tipo & Hooked end & Monofilamento \\
\hline Longitud & $35 \mathrm{~mm}$ & $50 \mathrm{~mm}$ \\
\hline Sección & $\varnothing=0,54 \mathrm{~mm}$ & $1.5 \times 0,4 \mathrm{~mm}$ \\
\hline Absorción & Ninguna & Ninguna \\
\hline Densidad específica & $7,8 \mathrm{gr} / \mathrm{cm}^{3}$ & $0,92 \mathrm{gr} / \mathrm{cm}^{3}$ \\
\hline Resistencia a tracción & $1150 \mathrm{MPa}$ & $550 \mathrm{MPa}$ \\
\hline Módulo de elasticidad & $210 \mathrm{GPa}$ & $4,3 \mathrm{GPa}$ \\
\hline
\end{tabular}

TABLA II. DOSIFICACIÓN DEL HORMIGÓN.

\begin{tabular}{|l|c|c|c|}
\hline \multicolumn{1}{|c|}{ Dosificación } & HPC & HPRFM & HPRFP \\
\hline Cemento & $370 \mathrm{~kg} / \mathrm{m}^{3}$ & $370 \mathrm{~kg} / \mathrm{m}^{3}$ & $370 \mathrm{~kg} / \mathrm{m}^{3}$ \\
\hline Agua & $170 \mathrm{~kg} / \mathrm{m}^{3}$ & $170 \mathrm{~kg} / \mathrm{m}^{3}$ & $170 \mathrm{~kg} / \mathrm{m}^{3}$ \\
\hline Microsílice & $30 \mathrm{~kg} / \mathrm{m}^{3}$ & $30 \mathrm{~kg} / \mathrm{m}^{3}$ & $30 \mathrm{~kg} / \mathrm{m}^{3}$ \\
\hline Arena $0-4 \mathrm{~mm}$ & $1215 \mathrm{~kg} / \mathrm{m}^{3}$ & $1215 \mathrm{~kg} / \mathrm{m}^{3}$ & $1215 \mathrm{~kg} / \mathrm{m}^{3}$ \\
\hline Grava $5-12 \mathrm{~mm}$ & $550 \mathrm{~kg} / \mathrm{m}^{3}$ & $550 \mathrm{~kg} / \mathrm{m}^{3}$ & $550 \mathrm{~kg} / \mathrm{m}^{3}$ \\
\hline $\begin{array}{l}\text { Superplastificante } \\
\text { (policarboxílico) }\end{array}$ & $1,7 \%$ & $1,7 \%$ & $1,7 \%$ \\
\hline Fibra & $6,3 \mathrm{~kg} / \mathrm{m}^{3}$ & $6,3 \mathrm{~kg} / \mathrm{m}^{3}$ & $6,3 \mathrm{~kg} / \mathrm{m}^{3}$ \\
\hline
\end{tabular}

Las mezclas se han fabricado en planta de hormigón con transporte en camión cuba hasta el punto de proyección. El equipo de proyección estaba constituido por una bomba de hormigón Putzmeister modelo BSA 1002, con capacidad de bombeo de hasta $20 \mathrm{~m}^{3} /$ hora, y un compresor de 8000 litros.

\subsection{Elementos y metodología de ensayo}

Se proyectaron paneles trapezoidales de dimensiones 600x600x150 $\mathrm{mm}$ para la extracción de testigos y paneles 600×600×100 mm y circulares de $800 \mathrm{~mm}$ de diámetro y $75 \mathrm{~mm}$ de espesor para los ensayos de tenacidad según EFNARC y Round Determinate Panel Test respectivamente. Previa a la ejecución de la proyección se determinó la consistencia del hormigón fresco mediante ensayo de cono de Abrams.

Los testigos se han extraído paralelamente a la dirección de proyección a la edad de 7 días, tal y como recomienda la norma UNE 83605 , realizándose un curado a $20 \pm 1^{\circ} \mathrm{C}$ y a $100 \%$ de humedad relativa hasta la fecha de realización de ensayo. Sus dimensiones aproximadas son $93 \mathrm{~mm}$ de diámetro y 150-140 mm de altura. Con los testigos se ha determinado densidad, porosidad, resistencia a compresión, módulo de elasticidad longitudinal y retracción por secado.

La tenacidad se ha evaluado siguiendo dos procedimientos distintos, el Plate Bending Test y el Round Determinate Panel Test.

En el Plate Bending Test de la norma europea EFNARC se aplica una carga centrada sobre el panel de dimensiones 600×600×100 mm apoyado en sus cuatro lados, en un ancho de $50 \mathrm{~mm}$, sobre un soporte rígido, registrándose la flecha resultante en el centro de panel (figura 2).

El Round Determinate Test es un ensayo sobre panel circular de $800 \mathrm{~mm}$ de diámetro y $75 \mathrm{~mm}$ de espesor apoyado en tres puntos pivotantes situados equidistantes y circunscritos en un diámetro de

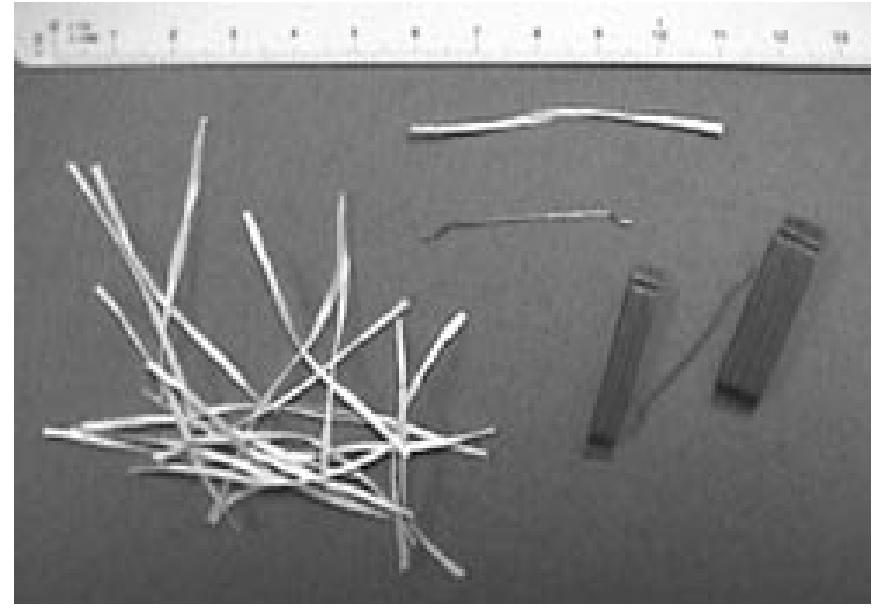

Figura 1. Fibra metálica y fibra polimérica estructural.

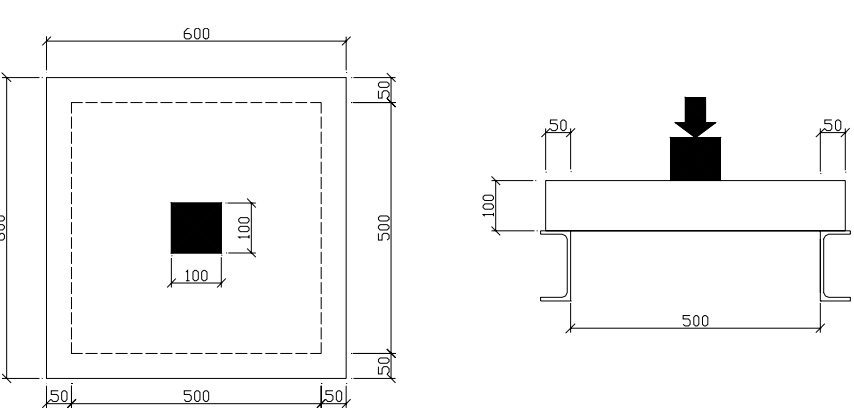

Figura 2. Plate Bending Test.

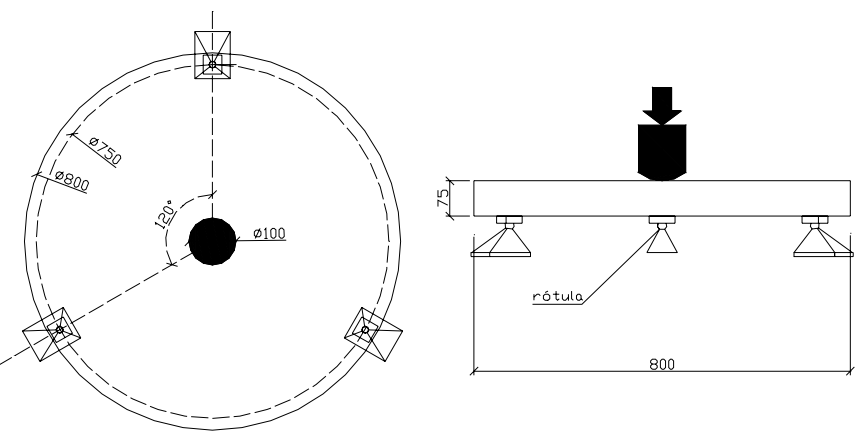

Figura 3. Round Determinate Test.

$750 \mathrm{~mm}$ (figura 3), al panel se le aplica una carga centrada mediante un plato circular de $100 \mathrm{~mm}$ de diámetro determinándose la flecha resultante en el centro de panel.

De la representación gráfica carga-flecha del ensayo de placa (rectangular o circular) se determina la capacidad de absorción de energía definida como el área encerrada entre la curva y el eje de abscisas, estableciendo la tenacidad para un cierto nivel de deflexión: $25 \mathrm{~mm}$ en el Plate Bending Test y $40 \mathrm{~mm}$ en el Round Determinate Panel Test. Otra propiedad significativa es el fenómeno de hardening, entendido aquí como la capacidad del hormigón de incrementar carga una vez fisurado, y caracterizado por la carga máxima alcanzada después del último valle de una curva tipo carga-flecha. 


\section{RESULTADOS}

\subsection{Caracterización del hormigón}

En la Tabla III se detallan los resultados obtenidos en la caracterización de los tres hormigones. Con respecto al hormigón fresco existe una mayor pérdida de trabajabilidad del HPRFP con respecto al HPRFM. La caracterización del hormigón endurecido no muestra, como era de esperar, diferencias significativas entre los tres hormigones en cuanto a resistencia y módulo a compresión, densidad y porosidad. Únicamente se observa una menor eficacia de las fibras poliméricas con respecto a las metálicas frente a retracción.

Tabla III. Caracterización del hormigón.

\begin{tabular}{|l|c|c|c|}
\hline Ensayo & HPC & HPRFM & HPRFP \\
\hline Consistencia hormigón fresco & $22 \mathrm{~cm}$ & $21 \mathrm{~cm}$ & $19 \mathrm{~cm}$ \\
\hline Resistencia a compresión a 28 días & $33,4 \mathrm{MPa}$ & $36,0 \mathrm{MPa}$ & $33,9 \mathrm{MPa}$ \\
\hline Módulo de elasticidad a 28 días & $31,0 \mathrm{GPa}$ & $30,5 \mathrm{GPa}$ & $29,0 \mathrm{GPa}$ \\
\hline Densidad muestra seca & $2,18 \mathrm{~kg} / \mathrm{m}^{3}$ & $2,18 \mathrm{~kg} / \mathrm{m}^{3}$ & $2,18 \mathrm{~kg} / \mathrm{m}^{3}$ \\
\hline $\begin{array}{l}\text { Densidad muestra saturada } \\
\text { superficie seca }\end{array}$ & $2,31 \mathrm{~kg} / \mathrm{m}^{3}$ & $2,31 \mathrm{~kg} / \mathrm{m}^{3}$ & $2,31 \mathrm{~kg} / \mathrm{m}^{3}$ \\
\hline Porosidad & $13 \%$ & $13 \%$ & $13 \%$ \\
\hline Retracción (220 días) & $0,78 \mathrm{~mm} / \mathrm{m}$ & $0,62 \mathrm{~mm} / \mathrm{m}$ & $0,75 \mathrm{~mm} / \mathrm{m}$ \\
\hline
\end{tabular}

\subsection{Ensayos sobre placa}

\subsubsection{PLATE BENDING TEST (EFNARC TEST)}

En la Tabla IV se detallan los resultados obtenidos en los ensayos de placa EFNARC: absorción de energía a $25 \mathrm{~mm}$ de flecha $\left(E_{25}\right)$, carga máxima de hardening $\left(Q_{h a r d}\right)$ y flecha en la que se produce $\left(\mathrm{f}_{\text {hard }}\right)$, carga media a $25 \mathrm{~mm}$ de flecha $\left(Q_{25}\right)$, así como los cocientes $Q_{25} / Q_{\text {hard }}$ y $\left(Q_{25}-Q_{\text {hard }}\right) /\left(25-f_{\text {hard }}\right)$, que ilustran en términos relativos una idea de la capacidad de mantener carga del hormigón.

Tabla IV. Resultados ensayo EFNARC.

\begin{tabular}{|c|c|c|c|c|c|c|}
\hline Párametro & \multicolumn{2}{|c|}{ HPC } & \multicolumn{2}{|c|}{ HPRFM } & \multicolumn{2}{|c|}{ HPRFP } \\
\hline \multirow{3}{*}{$E_{25}(J)$} & $25^{*}$ & \multirow{3}{*}{22} & 899 & \multirow{3}{*}{801} & 666 & \multirow{3}{*}{647} \\
\hline & $18^{*}$ & & 734 & & 697 & \\
\hline & $23^{*}$ & & 771 & & 578 & \\
\hline \multirow{3}{*}{$\begin{array}{l}\mathrm{Q}_{\text {hard }}(\mathrm{N}) \\
\mathrm{f}_{\text {hard }}(\mathrm{mm})\end{array}$} & $* * *$ & \multirow{3}{*}{ *** } & $\begin{array}{c}48,67 \\
4,95\end{array}$ & \multirow{3}{*}{$\begin{array}{c}43,19 \\
5,7\end{array}$} & $\begin{array}{c}32,72 \\
7,32\end{array}$ & \multirow{3}{*}{$\begin{array}{c}32,50 \\
8,02\end{array}$} \\
\hline & $* * *$ & & $\begin{array}{c}40,91 \\
6,76\end{array}$ & & $\begin{array}{l}34,45 \\
10,03\end{array}$ & \\
\hline & $* * *$ & & $\begin{array}{c}39,99 \\
5,4\end{array}$ & & $\begin{array}{c}30,34 \\
6,71\end{array}$ & \\
\hline $\mathrm{Q}_{25}(\mathrm{~N})$ & \multicolumn{2}{|c|}{$* * *$} & \multicolumn{2}{|c|}{21,03} & \multicolumn{2}{|c|}{20,61} \\
\hline $\mathrm{Q}_{25} / \mathrm{Q}_{\text {hard }}$ & \multicolumn{2}{|c|}{$* * *$} & \multicolumn{2}{|c|}{0,492} & \multicolumn{2}{|c|}{0,632} \\
\hline$\left(\mathrm{Q}_{25}-\mathrm{Q}_{\text {hard }}\right) /\left(25-\mathrm{f}_{\text {hard }}\right)$ & \multicolumn{2}{|c|}{ *** } & \multicolumn{2}{|c|}{$-1,15$} & \multicolumn{2}{|c|}{-0.70} \\
\hline
\end{tabular}

*Valores de energía en el momento de rotura

En la figura 4 se muestran los comportamientos de los hormigones reforzados representando las gráficas carga-flecha y absorción de energíaflecha obtenida de la integración de la anterior.

Aunque se han alcanzado niveles de absorción de energía distintos, hay que tener en cuenta que ésta es función de la dosificación de fibra utilizada. De los resultados obtenidos se desprende un mejor comportamiento de la fibra polimérica de alto módulo con respecto a
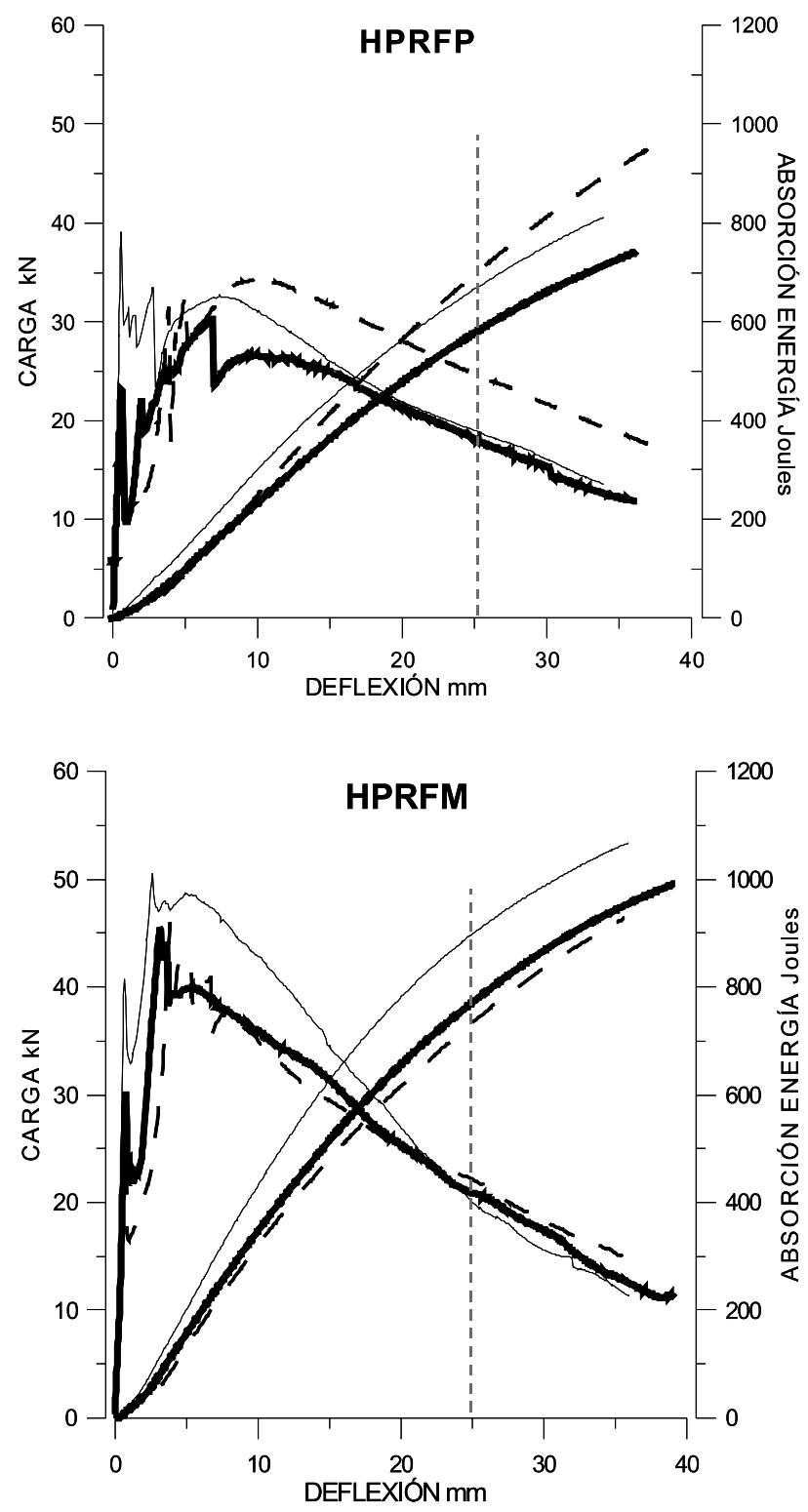

Figura 4. Plate Bending Test. Curvas carga-flecha y absorción de energía-flecha.

la metálica a medida que incrementa la flecha; ello se manifiesta por una ligera mejor capacidad de mantener carga en el HPRFP que en el HPRFM con cociente $Q_{25} / Q_{\text {hard }}$ más alto y pendiente $\left(Q_{25}-Q_{\text {hard }}\right) /\left(25-f_{\text {hard }}\right)$ menor. En este sentido destaca la consecución de la carga máxima de hardening a valores de flecha superiores en el HPRFP.

\subsubsection{ROUND DETERMINATE PANEL TEST}

Los resultados obtenidos en los ensayos sobre placa circular se presentan en la Tabla V. De forma análoga que en el EFNARC Test, se ha determinado la absorción de energía para una deflexión de 40 $\mathrm{mm}\left(E_{40}\right)$, carga máxima de hardening $\left(Q_{\text {hard }}\right)$ y flecha en la que se produce $\left(\mathrm{f}_{\text {hard }}\right)$, carga media a $40 \mathrm{~mm}$ de flecha $\left(Q_{40}\right)$, así como el cociente $Q_{40} / Q_{\text {hard }} \mathrm{y}\left(Q_{40}-Q_{\text {hard }}\right) /\left(40-f_{\text {hard }}\right)$, incluyendo, en este caso, la cargas alcanzadas $\left(Q_{\max }\right)$. En este caso se ha de destacar la rotura de paneles en 
dos fragmentos por lo que ambos se desestimaron en el cálculo de los valores medios tal y como recomienda la norma de ensayo. Asimismo en la figura 5 se han representado las curvas carga-flecha y absorción de energía-flecha de los paneles fragmentados en tres partes.

Tabla V. Resultados ensayo Round PanelTest.

\begin{tabular}{|c|c|c|c|c|c|c|}
\hline Párametro & \multicolumn{2}{|c|}{ HPC } & \multicolumn{2}{|c|}{ HPRFM } & \multicolumn{2}{|c|}{ HPRFP } \\
\hline \multirow{3}{*}{$\mathrm{E}_{40}(\mathrm{~J})$} & $10^{* *}$ & \multirow{3}{*}{10} & $430^{\prime}$ & \multirow{3}{*}{390} & 258 & \multirow{3}{*}{258} \\
\hline & $9^{* *}$ & & 360 & & 259 & \\
\hline & $7^{*}$ & & $290^{*}$ & & $214^{*}$ & \\
\hline \multirow{2}{*}{$\begin{array}{l}\mathrm{Q}_{\max }(\mathrm{N}) \\
\mathrm{f}_{\mathrm{Q} \max }(\mathrm{mm})\end{array}$} & $\begin{array}{c}21,56 \\
0,42\end{array}$ & \multirow{2}{*}{$\begin{array}{c}22,04 \\
0,39\end{array}$} & $\begin{array}{c}24,01 \\
0,47\end{array}$ & \multirow{2}{*}{$\begin{array}{c}23,49 \\
0,44\end{array}$} & $\begin{array}{l}22,99 \mathrm{~N} \\
0,43 \mathrm{~mm}\end{array}$ & \multirow{2}{*}{$\begin{array}{c}24,02 \\
0,41\end{array}$} \\
\hline & $\begin{array}{c}22,52 \\
0,36\end{array}$ & & $\begin{array}{c}22,88 \\
0,41\end{array}$ & & $\begin{array}{r}25,41 \mathrm{~N} \\
0,38 \mathrm{~mm}\end{array}$ & \\
\hline \multirow{2}{*}{$\begin{array}{l}\mathrm{Q}_{\text {hard }}(\mathrm{N}) \\
\mathrm{f}_{\text {hard }}(\mathrm{mm})\end{array}$} & $* * *$ & \multirow{2}{*}{$* * *$} & $\begin{array}{c}16,52 \\
2,92\end{array}$ & \multirow{2}{*}{$\begin{array}{c}16,53 \\
2,3\end{array}$} & $\begin{array}{c}8,91 \mathrm{~N} \\
8,64 \mathrm{~mm}\end{array}$ & \multirow{2}{*}{$\begin{array}{l}9,03 \\
8,97\end{array}$} \\
\hline & $* * *$ & & $\begin{array}{c}16,55 \\
1,62\end{array}$ & & $\begin{array}{r}9,14 \mathrm{~N} \\
9,3 \mathrm{~mm}\end{array}$ & \\
\hline $\mathrm{Q}_{40}(\mathrm{~N})$ & \multicolumn{2}{|c|}{$* * *$} & \multicolumn{2}{|c|}{4,90} & \multicolumn{2}{|c|}{3,54} \\
\hline $\mathrm{Q}_{40} / \mathrm{Q}_{\text {hard }}$ & \multicolumn{2}{|c|}{$* * *$} & \multicolumn{2}{|c|}{0,296} & \multicolumn{2}{|c|}{0,392} \\
\hline$\left(\mathrm{Q}_{40}-\mathrm{Q}_{\text {hard }}\right) /\left(40-\mathrm{f}_{\text {hard }}\right)$ & \multicolumn{2}{|c|}{$* * *$} & \multicolumn{2}{|c|}{$-0,308$} & \multicolumn{2}{|c|}{$-0,177$} \\
\hline
\end{tabular}

** Valores de energía en el momento de rotura.

De forma similar al ensayo EFNARC los distintos valores de absorción de energía obtenidos nos indica que las cantidades de fibra utilizadas no son equivalentes. Asimismo, se observa un comportamiento similar del HPRFP y HPRFM al presentado en el ensayo de placa cuadrada, con cargas máximas de hardening registradas en el HPRFP a flechas más altas que en el HPRFM y con una disminución de la carga de post-pico más gradual (suave). Con respecto a los valores de carga máxima se observa la nula influencia sobre ésta de la incorporación de la fibra.

\section{CONCLUSIONES}

Para las cantidades y tipos de fibra analizadas, la incorporación de fibras poliméricas de alto módulo en el hormigón proyectado aporta al material gran capacidad de carga después de fisuración, llegando a niveles de tenacidad similares que en hormigones proyectados reforzados con fibra metálica. No obstante se observa mejor comportamiento del HPRFP valores de flecha altos, por el contrario, el HPRFM se ha mostrado más eficaz a valores de flecha bajos.

Con respecto al tipo de ensayo, tanto el Round Panel Test, como el Plate Bending Test se han mostrado eficaces para la evaluación de la tenacidad de hormigones reforzados con fibra. En este sentido parece más recomendable, por su mayor sencillez, el ensayo de placa circular para el control, mientras que el EFNARC Test se muestra más adecuado para estudios previos, al reproducir mejor el comportamiento in situ del hormigón proyectado como material estructural.
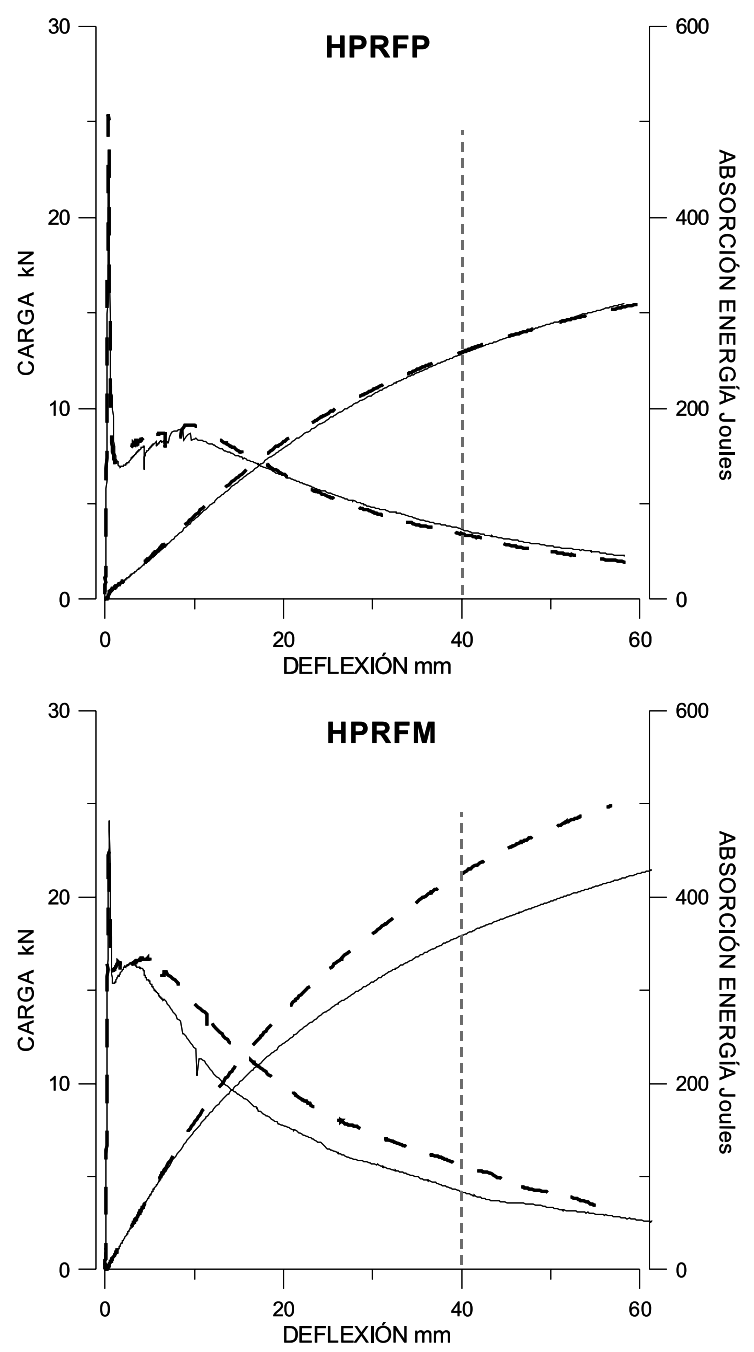

Figura 5. Round Planel Test. Curvas carga-flecha y absorción de energía-flecha.

\section{REFERENCIAS}

1. N. Banthia, P. Gupta, C. Yan and D. R. Morgan, "How tough is fiber reinforced shotcrete? Part 1, beam test." Concrete International. June 1999.

2. N. Banthia, P. Gupta, C. Yan and D. R. Morgan, "How tough is fiber reinforced shotcrete? Part 2, plate test". Concrete International. August 1999.

3. D. R. Morgan, R. Heere, N. McAskill and C. Chan, “Comparative evaluation of system ductility of mesh and fibre reinforced shotcretes". Shotcrete for underground support "European Specification for Sprayed Concrete".

4. European Federation of National Associations of Specialist Contractors and Material Suppliers for the Construction Industry (EFNARC )1996.VIII. April 1999.

5. E. S. Bernard, "Correlations in the performance of fibre reinforced shotcrete beam and panels". Civil Engineering report CE9, School of Civic Engineering and Environment, UWS Nepean, July 1999.

6. T. Garcia, L. Agulló, A. Aguado, J. L. Rodríguez, "Propuesta metodológica para dosificación del hormigón proyectado". Hormigón y acero n²20, segundo trimestre 2001 .

Recibido: 1.2 .03

Aceptado: 30.11 .03 